\title{
La economía rural en Chile: entre la pobreza y el desarrollo
}

\author{
EDGARDO GUILLERMO OYARZUN MÉNDEZ \\ Instituto de Turismo, UNIVERSIDAD AUSTRAL DE CHILE, CHILE. E-mail: \\ eoyarzun@uach.cl
}

\section{BELÉN MIRANDA ESCOLAR}

Departamento de Economía Aplicada, UNIVERSIDAD DE VALLADOLID, ESPAÑA. Email: belen@eco.uva.es

\section{RESUMEN}

El trabajo ofrece la visión de la economía rural chilena. Una realidad dicotómica que se mueve entre un medio rural diversificado (turismo, frutales, madera, pesca y vino, fundamentalmente), innovador, que exporta, dominado por empresas transnacionales, y la pequeña agricultura campesina, que se asocia con elevados índices de pobreza y que, en estos momentos, se encuentra ante un futuro incierto. Es hacia este sector de la población rural hacia donde deben dirigirse determinadas políticas de desarrollo encaminadas a lograr la reducción de la pobreza.

Palabras clave: Desarrollo rural, pobreza, agricultura, turismo rural.

\section{The Rural Economy in Chile: Between Poverty and Development}

\begin{abstract}
The paper offers a view of rural economy in Chile. A dichotomical reality between a rural diversified sector (tourism, fruit trees, wood, fishing and wine, mainly), innovator, exporter, under control by transnational enterprises, and the short farming agriculture which shows high levels of poverty and it has an uncertain future at this moment. Specific development policies must be implemented to affect this kind of rural population and to reduce poverty.
\end{abstract}

Keywords: Rural development, poverty, agriculture, rural tourism.

Clasificación JEL: O13, O18, Q26, R58

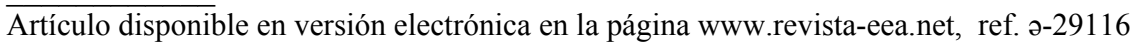




\section{INTRODUCCIÓN}

La realidad del medio rural chileno se manifiesta de forma dicotómica. Por un lado, un sector productor dominado por empresas transnacionales (frutícola, que ha sido fundamental en la diversificación de la base exportadora del país; vitivinícola; forestal; $y$, pesquero, fundamentalmente), con moderna tecnología y capacidad de innovación. Por otro, los pequeños propietarios, inmersos en una agricultura de subsistencia, con escaso capital y sin tecnología. Es la agricultura campesina o familiar que tiene su origen en el campo y mantiene una relación hombre-tierra muy estrecha, con características culturales y sociales bien definidas. Es este segmento el que se asocia con los índices de pobreza de las zonas rurales y el que se encuentra ante un futuro incierto y cada vez más constreñido.

En este trabajo deseamos poner de manifiesto las dos formas de producción agrícola claramente diferenciadas en el medio rural chileno, que ponen de manifiesto la heterogeneidad existente que, en no pocas ocasiones, es fuente de conflictos. Asimismo, se analizarán las políticas instrumentadas desde los distintos poderes públicos para hacer más viable la convivencia entre ambos, teniendo en cuenta que la pobreza y la desigualdad que afectan más intensamente a las regiones con una mayor proporción de población rural se perfilan, a pesar de los logros obtenidos a lo largo de las dos últimas décadas, como un claro factor que limita las posibilidades de desarrollo de los espacios rurales del país.

\section{EL ESPACIO RURAL CHILENO}

Como es sabido, la delimitación del espacio rural ofrece más de una alternativa. En el caso de Chile, la más utilizada es la clasificación del Instituto Nacional de Estadística (INE), que define como localidad rural a "un asentamiento humano concentrado o disperso, cuya población no supera los 1000 habitantes, o entre 1.000 y 2.000 en caso que la población económicamente activa dedicada a las actividades secundarias o terciarias no supere el 50\%" (INE, 1992). Pero, más allá de los recursos naturales, materiales y del sistema productivo, interesa centrarse en la población residente en el territorio que se defina como rural. "El mundo rural se ha convertido en la periferia dentro de la creciente internacionalización de las economías de todos los países" (Blanco Portillo y Benayas del Álamo, 1994), incluso las decisiones que le afectan se toman en instancias muy distantes a él. Esta situación para Chile se ve más agravada, aún, por la fuerte centralización en torno al gobierno central y los gobiernos regionales, que se manifiesta en el diseño y ejecución de políticas y estrategias de desarrollo rural, en ocasiones poco adecuadas a los estrangulamientos que se desea afectar. 


\section{CARACTERIZACIÓN DE LA POBLACIÓN RURAL CHILENA}

De acuerdo con el último Censo de Población de Chile (2002), la población definida como rural alcanzaba al $13,4 \%$ del total nacional (Tabla 1) registrándose, en la última década, una caída de 3,1 puntos porcentuales ${ }^{1}$.

Tabla 1

Distribución de la población nacional por regiones (Censo 2002)

\begin{tabular}{|c|c|c|c|}
\hline \multirow{3}{*}{ Región } & \multicolumn{3}{|c|}{ Población } \\
\hline & \multicolumn{2}{|c|}{ Censo 2002} & \multirow{2}{*}{ Total } \\
\hline & Urbana & Rural & \\
\hline Total Chile & 13.090 .113 & 2.026.322 & 15.116 .435 \\
\hline Tarapacá & 403.138 & 25.456 & 428.594 \\
\hline Antofagasta & 482.546 & 11.438 & 493.984 \\
\hline Atacama & 232.619 & 21.717 & 254.336 \\
\hline Coquimbo & 470.922 & 132.288 & 603.210 \\
\hline Valparaíso & 1.409 .902 & 129.950 & 1.539 .852 \\
\hline General Bernardo O'Higgins & 548.584 & 232.043 & 780.627 \\
\hline Maule & 603.020 & 305.077 & 908.097 \\
\hline Bio-Bio & 1.529 .306 & 333.256 & 1.861 .562 \\
\hline Araucanía & 588.408 & 281.127 & 869.535 \\
\hline Los Lagos & 734.379 & 338.756 & 1.073 .135 \\
\hline Aysén del General Carlos Ibáñez del Campo & 73.607 & 17.885 & 91.492 \\
\hline Magallanes y la Antártica Chilena & 139.669 & 11.157 & 150.826 \\
\hline Metropolitana de Santiago & 5.875 .013 & 186.172 & 6.061 .185 \\
\hline
\end{tabular}

Fuente: Instituto Nacional de Estadísticas (INE).

En los últimos cuarenta años, Chile, al igual que la mayoría de los países iberoamericanos, ha vivido un proceso migratorio acelerado, un verdadero éxodo desde el campo a la ciudad. En algunas comunas rurales este proceso ha significado la disminución de más del $40 \%$ de la población en sólo una década, disminuyendo la densidad de población en algunas zonas rurales hasta niveles críticos. Tan sólo una rápida revisión de los datos censales que se recogen en la Tabla 2, desde el año 1865, deja patente esta cruda realidad. Si bien la disminución de la población en las áreas rurales es una tendencia que se produce a escala mundial, en el caso chileno se ve agravada por diferentes razones que deben tenerse en cuenta a la hora de llevar a cabo una política de desarrollo rural.

\footnotetext{
${ }^{1}$ En el anterior Censo de 1992, la población rural representaba el 16,5\%.
} 
Tabla 2

Evolución de la población urbana y rural en Chile. Datos censales 1865-2002

\begin{tabular}{|c|c|c|c|c|}
\hline \multirow{2}{*}{ Censos } & \multicolumn{2}{|c|}{ Población urbana } & \multicolumn{2}{c|}{ Población rural } \\
\cline { 2 - 5 } & Habitantes & Porcentaje & Habitantes & Porcentaje \\
\hline 1865 & 520.663 & 28,6 & 1.298 .560 & 71,6 \\
\hline 1875 & 725.545 & 34,9 & 1.350 .426 & 65,1 \\
\hline 1885 & 1.045 .655 & 40,2 & 1.461 .715 & 58,9 \\
\hline 1905 & 1.226 .435 & 45,5 & 1.469 .476 & 54,5 \\
\hline 1907 & 1.396 .912 & 43,2 & 1.834 .584 & 56,8 \\
\hline 1920 & 1.732 .567 & 46,4 & 1.999 .006 & 53,6 \\
\hline 1930 & 2.119 .221 & 49,4 & 2.168 .224 & 50,6 \\
\hline 1940 & 2.663 .479 & 52,4 & 2.390 .060 & 47,6 \\
\hline 1952 & 3.536 .878 & 59,6 & 2.393 .931 & 40,4 \\
\hline 2002 & 13.090 .113 & 86,6 & 2.026 .322 & 13,4 \\
\hline
\end{tabular}

Fuente: Elaboración propia a partir de los Censos del INE (Censos 1865-2002) y Errázuriz, citado por Huerta (1989).

Tabla 3

Miembros del hogar del productor agropecuario que trabajan en la explotación, por edad y sexo, según tamaño de las explotaciones.

\begin{tabular}{|c|c|c|c|c|c|c|}
\hline \multirow{3}{*}{ Tamaño de las Explotaciones } & \multirow{2}{*}{\multicolumn{2}{|c|}{$\begin{array}{l}\text { Explotaciones } \\
\text { Informantes }\end{array}$}} & \multicolumn{4}{|c|}{ Número de Personas del Hogar } \\
\hline & & & \multirow{2}{*}{ TOTAL } & \multicolumn{2}{|c|}{ De 15 años y más } & \multirow{2}{*}{$\begin{array}{c}\text { Menores } \\
\text { de } 15 \\
\text { años }\end{array}$} \\
\hline & Número & Superficie & & Hombres & Mujeres & \\
\hline Total país & 186.891 & $4.660 .921,80$ & 324.601 & 223.558 & 94.915 & 6.128 \\
\hline Explotaciones sin tierra & 1.936 & 0 & 2.797 & 1.903 & 806 & 88 \\
\hline Explotaciones con tierra & 184.955 & $4.660 .921,80$ & 321.804 & 221.655 & 94.109 & 6.040 \\
\hline Menores de 1 ha. & 26.575 & $13.060,70$ & 37.716 & 26.273 & 10.925 & 518 \\
\hline De 1 a menos de 5 ha. & 51.929 & $129.372,90$ & 84.103 & 55.664 & 26.647 & 1.792 \\
\hline De 5 a menos de 10 ha. & 31.733 & $225.445,70$ & 57.793 & 38.702 & 17.921 & 1.170 \\
\hline De 10 a menos de 20 ha. & 30.684 & $430.206,80$ & 58.444 & 39.922 & 17.342 & 1.180 \\
\hline De 20 a menos de 50 ha. & 26.686 & $826.356,90$ & 51.528 & 36.571 & 13.991 & 966 \\
\hline De 50 a menos de 100 ha. & 10.134 & $689.904,50$ & 19.362 & 14.491 & 4.593 & 278 \\
\hline De 100 a menos de 200 ha. & 4.308 & $586.527,90$ & 7.954 & 6.113 & 1.757 & 84 \\
\hline De 200 a menos de 500 ha. & 2.168 & $649.395,70$ & 3.686 & 2.908 & 740 & 38 \\
\hline De 500 a menos de 1.000 ha. & 497 & $336.841,50$ & 816 & 684 & 119 & 13 \\
\hline De 1.000 a menos de 2.000 ha. & 158 & $211.466,90$ & 239 & 204 & 35 & 0 \\
\hline De 2.000 ha. y más & 83 & $562.342,30$ & 163 & 123 & 39 & 1 \\
\hline
\end{tabular}

Fuente: INE: Censo Agropecuario. 
- La primera es que las condiciones de producción de un importante segmento de la población son precarias. Del total de las explotaciones agrarias existentes en el país, el 4,84\% tiene menos de 10 hectáreas de superficie, explotaciones que en su mayoría forman parte de una economía de subsistencia (Tabla 3).

- La segunda es el deterioro socio-espacial que se manifiesta en deficiencias de: accesibilidad, infraestructuras de salud, educación, agua potable, alcantarillado, comunicaciones, energía,... Ahora bien, debe reconocerse que en los diferentes gobiernos post-régimen militar se ha intensificado la lucha contra la pobreza, a través de proyectos de electrificación y agua potable rural, saneamiento de la propiedad de la tierra, y un importante número de programas más ${ }^{2}$.

- La tercera es la situación de pobreza e indigencia que viven muchas de las personas que habitan el medio rural chileno y que se manifiestan más agudamente que en las áreas urbanas. Los estudios realizados basados en la definición de líneas de pobreza ${ }^{3}$ indican que ésta afectaba, en 2009, a un total de 2.508 .880 personas, lo que representa el $15,13 \%$ de la población total chilena (Tabla 4). Si tomamos en consideración las comunas, de las 100 más pobres del país, 84 son rurales ${ }^{4}$.

- La cuarta y última es que aquellas regiones que concentran una mayor prprción de población rural, son también las que padecen con mayor intensidad el flagelo de la pobreza y la desigualdad. Este hecho se manifiesta de forma especial en las regiones del Maule (21\%), Araucanía (27\%) y Los Lagos (incluyendo Los Ríos) (16,2\%) (

- Tabla 4), y da cuenta de la existencia de un problema que, si bien se ha venido reduciendo a lo largo del tiempo, como se analizará posteriormente, aún se perfila como un claro factor que limita las posibilidades de desarrollo de los espacios rurales del país y se convierte en el principal desafío para las políticas que se instrumenten en este sentido.

${ }^{2}$ Al comenzar los gobiernos de la concertación se estimaba que había 5 millones de chilenos que vivían en situación de pobreza.

${ }^{3}$ Se está en situación de pobreza cuando el ingreso per capita del hogar es inferior a dos canastas básicas de alimentos en la zona urbana y a 1,75 en el medio rural. La indigencia se produce cuando el ingreso per capita del hogar es inferior a una canasta básica.

${ }^{4}$ Una comuna es la subdivisión de la provincia a efectos de la administración local, que tiene por objeto facilitar la prestación de servicios, para satisfacer los intereses locales y favorecer la organización y participación de la comunidad. En cada una el órgano de gobierno es la Municipalidad, bajo la responsabilidad de un Consejo Municipal encabezado por un Alcalde, elegido por votación popular. 
Tabla 4

Número de personas en relación con las líneas de pobreza según región. Año 2009

\begin{tabular}{|l|c|c|c|c|}
\hline \multirow{2}{*}{ Región } & \multicolumn{4}{|c|}{ Situación de Indigencia o Pobreza } \\
\cline { 2 - 5 } & Indigencia & Pobre no Indigente & No pobre & Total \\
\hline Tarapacá & 7.016 & 38.951 & 244.380 & 290.347 \\
\hline Antofagasta & 4.014 & 38.967 & 494.009 & 536.990 \\
\hline Atacama & 19.755 & 27.502 & 222.469 & 269.726 \\
\hline Coquimbo & 29.732 & 85.717 & 581.243 & 696.692 \\
\hline Valparaíso & 58.607 & 197.791 & 1.446 .996 & 1.703 .394 \\
\hline O`Higgins & 25.209 & 85.454 & 754.703 & 865.366 \\
\hline Maule & 48.375 & 156.167 & 781.502 & 986.044 \\
\hline Bío Bío & 102.599 & 314.703 & 1.572 .555 & 1.989 .857 \\
\hline Araucanía & 84.442 & 169.057 & 681.842 & 935.341 \\
\hline Los Lagos & 19.386 & 93.595 & 682.893 & 795.874 \\
\hline Aysen & 4.876 & 9.292 & 79.619 & 93.787 \\
\hline Magallanes & 4.402 & 8.876 & 133.207 & 146.485 \\
\hline Metropolitana & 181.493 & 595.523 & 5.952 .956 & 6.729 .972 \\
\hline Los Ríos & 22.275 & 52.187 & 290.068 & 364.530 \\
\hline Arica y Parinacota & 8.206 & 14.711 & 155.513 & 178.430 \\
\hline Total & $\mathbf{6 2 0 . 3 8 7}$ & $\mathbf{1 . 8 8 8 . 4 9 3}$ & $\mathbf{1 4 . 0 7 3 . 9 5 5}$ & $\mathbf{1 6 . 5 8 2 . 8 3 5}$ \\
\hline
\end{tabular}

Fuente: Ministerio de Planificación y Coordinación (MIDEPLAN) (2009): División Social. Encuesta CASEN.

Actualmente, la situación de la población rural ha comenzado a ser considerada por parte de las autoridades públicas, no sólo como un problema demográfico, sino, también, como un problema ambiental, debido al hiperpoblamiento de las grandes ciudades y las de tamaño intermedio, y como un problema geopolítico, puesto que muchas áreas limítrofes se hallan en franco retroceso poblacional y económico, lo que puede acarrear efectos muy negativos para el territorio. De hecho, estas preocupaciones han sido las que han justificado, en parte, la propuesta de estrategias que vinculan ocio y ruralidad.

Teóricamente, se debe dejar claro que la participación y la existencia de un capital social sólido constituye la "piedra angular" del desarrollo. Sin embargo, la realidad es que la opinión de los campesinos pobres pocas veces es tenida en cuenta en los procesos de planificación, y menos aún a la hora de asignarles un papel en las actividades de desarrollo que se promueven. Este hecho se explica porque los pobres carecen de una estructura organizativa fuerte que, efectivamente, pueda articular sus necesidades y sirva a sus intereses.

Marginados, y con frecuencia sometidos a condiciones de explotación, no cuentan con los medios para obtener un mayor acceso a los recursos y a los 
servicios que podrían ayudarles a mejorar sus condiciones de vida. A menos que a los pobres les sean proporcionadas las herramientas para participar plenamente en el desarrollo, continuarán siendo excluidos de los beneficios éste. Como consecuencia, no serán capaces de contribuir con todo su potencial a la expansión de los mercados rurales, con ahorro e inversiones, todos ellos elementos de gran relevancia en cualquier proceso de desarrollo rural (Galán, 1994).

Como señala Hernández Sánchez (1989), la población es el protagonista y a la vez el destinatario del desarrollo. Cualquier programa o proyecto encaminado a este fin, debe elaborarse sobre la base de conocer quiénes serán los sujetos de su acción, por lo que establecer un perfil básico de la "población objetivo" es el primer paso a seguir. No se pretende en este trabajo cumplir minuciosamente con ello, pero sí se proporcionará información suficiente que permita aproximarnos a una caracterización del habitante rural chileno.

\section{POBREZA, RURALIDAD Y AGRICULTURA}

Como ya se ha señalado, la pobreza en Chile se ha reducido significativamente en las últimas dos décadas, pasando de un $38,6 \%$ de la población total, en 1990, a un 18,8\% en el año 2003 (Alvayay; Rojas; y, Villarroel, 2004), y a un $15,1 \%$ en 2009. Sin embargo, al diferenciar entre las áreas rural y urbana, se observa una mayor incidencia de la pobreza en el medio rural, hasta el año 2009 en el que esta situación revierte, dado que este porcentaje disminuyó en las zonas urbanas hasta el 15,4\%, haciéndolo en las zonas rurales del país hasta el 12,9\% (Tabla 5).

Tabla 5

Incidencia de la Pobreza ${ }^{1}$ según zona. (En porcentaje)

\begin{tabular}{|c|c|c|c|c|c|c|c|c|}
\hline Zona & 1990 & 1992 & 1994 & 1996 & 1998 & 2000 & $\mathbf{2 0 0 3}$ & $\mathbf{2 0 0 9}$ \\
\hline Urbana & 38,4 & 32,4 & 26,9 & 21,8 & 20,7 & 20,1 & 18,6 & 15,4 \\
\hline Rural & 39,5 & 33,4 & 30,9 & 30,6 & 27,6 & 23,8 & 20,1 & 12,9 \\
\hline
\end{tabular}

${ }^{1}$ Pobreza incluye indigencia.

Fuente: Elaboración propia a partir de la Encuesta CASEN.

El medio rural, por su parte, también ha mostrado cambios en este último período, siendo uno de los más notables el fuerte proceso de des-ruralización que se evidencia. El Censo de 2002, registraba un 13,4\% de población rural, lo que contrasta con el nivel de ruralidad del país de hace 40 años, que alcanzaba al $40 \%$. Cifras recientes señalan que este proceso de des-ruralización del país 
continúa, advirtiéndose una disminución de la población rural de un $8,2 \%$ en la última década.

Otro aspecto importante que debe destacarse es que la ruralidad no es homogénea a lo largo de todo Chile, siendo más significativa en zonas del centro y el sur, que presentan una mayor concentración de tierras de uso agrícola. En algunas de estas regiones el porcentaje de la población rural supera el $30 \%$.

Los datos correspondientes al medio rural chileno muestran, además, cambios en las condiciones de vida de las familias que influyen en las características de la pobreza rural. Debe mencionarse, por ejemplo, que la población mayor de 10 años ha mejorado el índice de alfabetización, pasando de un $85,97 \%$ en 1992 , a un $89,2 \%$ en 2002 . Estos datos reflejan el resultado de la política educativa que, en la última década, ha ampliado la cobertura de la enseñanza básica, además de realizar un importante esfuerzo en materia de infraestructura educacional.

Adicionalmente, en la actualidad casi un $90 \%$ de las viviendas ubicadas en las zonas rurales del país son casas; al menos el $81,7 \%$ de estas viviendas tienen una fuente de abastecimiento de agua segura (red pública o pozos) y el $83,7 \%$ se abastece de electricidad por medio de la red pública. En materia de telecomunicaciones, la política de acceso universal implantada en el país, con especial énfasis en el desarrollo de la telefonía rural, ha extendido la infraestructura básica a lo largo del territorio nacional. Esto ha permitido elevar la cobertura de acceso a través de teléfonos públicos a prácticamente todo el territorio nacional y disponer del servicio de telefonía móvil en el $41 \%$ de los hogares rurales. Progresos sustantivos se han materializado, también, en el ámbito de las obras públicas, incluyendo carreteras y transporte, lo que junto a los avances en telecomunicaciones, ha hecho posible alcanzar mayores niveles de interconexión física y virtual del país durante la última década.

Respecto a la actividad económica, si bien sabemos que "la ruralidad" no es sinónimo de "lo agrario", la producción primaria agrícola sigue representando la principal actividad económica del medio rural. En efecto, más del $60 \%$ de la población rural depende directamente de la actividad agrícola primaria; a ello se debe añadir su impacto indirecto sobre las restantes actividades económicas locales. Sin embargo, se observan dos fenómenos importantes.

- El primero, es que la incidencia de la actividad agrícola no es homogénea en las distintas regiones y comunas del país, existiendo, en algunos casos, una alta dependencia entre la ésta y la actividad económica de toda una localidad.

- El segundo, es que ha ido aumentando la importancia del ingreso rural no 
agrícola $^{5}$ en las familias rurales más pobres, tanto en el conjunto de América Latina como en Chile, familias en las que se concentra alrededor del 40\% del ingreso rural total (Berdegué; Reardon; y, Escobar, 2003).

En conclusión, los menores niveles de pobreza y los cambios que se evidencian en el espacio rural, nos enfrentan a un nuevo escenario para el desarrollo rural chileno. Las políticas sociales y económicas han cumplido su rol y han generado importantes resultados, pero requieren ser revisadas desde este nuevo contexto, de modo que considere estos cambios y se adapte a ellos para seguir avanzando en contribuir a la superación de la pobreza.

\section{EL MERCADO DE TRABAJO EN EL ESPACIO RURAL CHILENO}

Tradicionalmente el medio rural se ha caracterizado por sustentar su economía en actividades del sector primario: agricultura, silvicultura, ganadería, pesca artesanal, y extracción y recolección de mariscos; todas ellas actividades de carácter extractivo. La identificación del medio rural con la tierra (agricultura, silvicultura, ganadería,...) es fuerte, pero, sin embargo, en los espacios rurales del litoral marítimo, tan importantes en un país como Chile, son las actividades relacionadas con el mar (pesca, extracción de mariscos, recolección de algas,...) las que prevalecen. Sin embargo, su importancia como actividad generadora de empleo ha ido disminuyendo drásticamente en todos los países a medida que se van alcanzado mayores niveles de desarrollo económico.

Como ya se ha señalado, en la agricultura chilena se distinguen dos grandes modalidades de estructura productiva con características bien diferenciadas: la empresarial y la campesina, siendo la singularidad más importante de esta última el empleo de fuerza de trabajo familiar, mientras que la primera utiliza mano de obra asalariada. Pero no es ésta la única diferencia entre ambas. La Tabla 6 recoge de forma resumida los principales elementos diferenciales.

${ }^{5}$ Aquel ingreso generado por habitantes rurales a través del autoempleo o el trabajo asalariado en los sectores secundario y terciario de la economía. 
Tabla 6

Características diferenciales de las agriculturas campesina y empresarial

\begin{tabular}{|l|l|l|}
\hline \multicolumn{1}{|c|}{ Atributos } & \multicolumn{1}{|c|}{ Agricultura campesina } & \multicolumn{1}{c|}{ Agricultura empresarial } \\
\hline $\begin{array}{l}\text { Objetivo de la } \\
\text { producción }\end{array}$ & $\begin{array}{l}\text { Reproducción de la familia y de la unidad } \\
\text { de producción }\end{array}$ & $\begin{array}{l}\text { Maximizar la tasa de ganancia y } \\
\text { la acumulación de capital }\end{array}$ \\
\hline $\begin{array}{l}\text { Origen de la fuerza } \\
\text { de trabajo }\end{array}$ & $\begin{array}{l}\text { Fundamentalmente familiar y, en } \\
\text { ocasiones, intercambio recíproco con } \\
\text { otras unidades; excepcionalmente } \\
\text { asalariada en cantidades marginales }\end{array}$ & Asalariada \\
\hline $\begin{array}{l}\text { Compromiso laboral } \\
\text { del jefe con la mano } \\
\text { de obra }\end{array}$ & Absoluto & $\begin{array}{l}\text { Inexistente, salvo por obligación } \\
\text { legal }\end{array}$ \\
\hline $\begin{array}{l}\text { Tecnología } \\
\text { Alta intensidad de mano de obra, baja } \\
\text { densidad de "capital y de insumos } \\
\text { comprados por jornada de trabajo }\end{array}$ & $\begin{array}{l}\text { Mayor densidad de capital por } \\
\text { activo y mayor proporción de } \\
\text { insumos comprados en el valor } \\
\text { del producto final }\end{array}$ \\
\hline $\begin{array}{l}\text { Destino del } \\
\text { producto y origen de } \\
\text { los insumos }\end{array}$ & Parcialmente mercantil & Mercantil \\
\hline $\begin{array}{l}\text { Criterio de } \\
\text { intensificación de } \\
\text { trabajo }\end{array}$ & $\begin{array}{l}\text { Máximo producto total, aún a costa del } \\
\text { descenso del producto medio. Limite: } \\
\text { producto marginal cero }\end{array}$ & $\begin{array}{l}\text { Productividad marginal mayor } \\
\text { que el salario }\end{array}$ \\
\hline $\begin{array}{l}\text { Riesgo e } \\
\text { incertidumbre }\end{array}$ & $\begin{array}{l}\text { Evasión no probabilística: "algoritmo de } \\
\text { supervivencia" }\end{array}$ & $\begin{array}{l}\text { Internalización probabilística } \\
\text { buscando tasas de ganancia } \\
\text { proporcionales al riesgo }\end{array}$ \\
\hline $\begin{array}{l}\text { Carácter de la } \\
\text { fuerza de trabajo }\end{array}$ & $\begin{array}{l}\text { Fuerza valorizada de trabajo intransferible } \\
\text { o marginal }\end{array}$ & $\begin{array}{l}\text { Sólo emplea fuerza de trabajo } \\
\text { transferible en función de } \\
\text { calificación. }\end{array}$ \\
\hline $\begin{array}{l}\text { Componentes del } \\
\text { ingreso o producto } \\
\text { neto }\end{array}$ & $\begin{array}{l}\text { Producto o ingreso familiar indivisible y } \\
\text { realizado parcialmente en especie }\end{array}$ & $\begin{array}{l}\text { Salario, renta y ganancias, } \\
\text { exclusivamente pecuniarias. }\end{array}$ \\
\hline
\end{tabular}

Fuente: Adaptado de Schejtman (1996).

Sin embargo, en el subsector campesino, está disminuyendo la fuerza de trabajo familiar por su incapacidad para generar ingresos. Los recursos naturales que controla no crecen y, peor aún, se reducen, se fraccionan y se deterioran. Las regiones del sur del país son las que concentran el trabajo de carácter familiar. En las regiones del centro, marcadamente frutícolas, su impacto relativo es menor (Tabla 7). En este caso, la mujer tiene como opción el trabajo de "temporera" en tareas de recolección y envasado de frutas. 
Tabla 7

Miembros del hogar que viven en la explotación, por edad y sexo

\begin{tabular}{|c|c|c|c|c|c|c|}
\hline \multirow{3}{*}{$\begin{array}{l}\text { Clasificación } \\
\text { Geográfica }\end{array}$} & \multirow{2}{*}{\multicolumn{2}{|c|}{ Explotaciones Informantes }} & \multicolumn{4}{|c|}{$\begin{array}{l}\text { Miembros del Hogar del Productor } \\
\text { Agropecuario (Número de Personas) }\end{array}$} \\
\hline & & & \multirow{2}{*}{ TOTAL } & \multicolumn{2}{|c|}{ De 15 años y más } & \multirow{2}{*}{$\begin{array}{l}\text { Menores } \\
\text { de } 15 \text { años }\end{array}$} \\
\hline & Número & Superficie (ha) & & Hombres & Mujeres & \\
\hline Total país & 275.933 & $29.762 .610,65$ & 833.940 & 323.817 & 295.438 & 214.685 \\
\hline I Región & 1.781 & $325.475,49$ & 0 & 0 & 0 & 0 \\
\hline II Región & 1.865 & $374.407,11$ & 2.075 & 835 & 768 & 472 \\
\hline III Región & 2.561 & $3.769 .753,47$ & 4.669 & 1.924 & 1.697 & 1.048 \\
\hline IV Región & 15.012 & $3.987 .344,56$ & 46.996 & 17.832 & 16.888 & 12.276 \\
\hline V Región & 15.236 & $1.114 .922,11$ & 47.178 & 18.765 & 17.294 & 11.119 \\
\hline VI Región & 22.678 & $1.130 .724,19$ & 83.530 & 33.743 & 31.061 & 18.726 \\
\hline VII Región & 38.057 & $1.891 .583,75$ & 98.281 & 39.548 & 35.666 & 23.067 \\
\hline VIII Región & 56.639 & $1.786 .379,16$ & 155.214 & 60.796 & 54.839 & 39.579 \\
\hline IX Región & 54.599 & $1.936 .798,95$ & 190.328 & 70.473 & 63.311 & 56.544 \\
\hline X Región & 33.635 & $2.523 .056,92$ & 162.413 & 62.409 & 57.957 & 42.047 \\
\hline XI Región & 3.221 & $3.251 .078,49$ & 0 & 0 & 0 & 0 \\
\hline XII Región & 1.298 & $5.356 .956,00$ & 0 & 0 & 0 & 0 \\
\hline Región Metropolitana & 11.216 & $1.133 .838,97$ & 43.256 & 17.492 & 15.957 & 9.807 \\
\hline XIV Región & 15.714 & $979.291,59$ & & & & \\
\hline XV Región & 2.421 & $201.000,19$ & & & & \\
\hline
\end{tabular}

Nota: Las celdas que aparecen con valor 0 se han obviado a efectos de esta investigación.

Fuente: INE (2007): Censo Agropecuario.

Se verifica que las formas de desarrollo que se están produciendo en el medio rural, ya sea espontánea y/o planificadamente, provocan cambios en la estructura productiva que modifican las características ocupacionales de la población, asumiendo roles cada vez más relevantes la mujer y los miembros más jóvenes del grupo familiar. En el contexto del desarrollo local, no todas las iniciativas surgen de procesos estructurados y perfectamente planificados. La informalidad es una de las variables que atenta muchas veces contra la viabilidad y sostenibilidad económica de algunos emprendimientos, situación que es más crítica si se considera que la condición de "formalidad" se exige para recibir el apoyo de los organismos gubernamentales responsables del fomento productivo. Hernández (1989) señala: "de hecho, como varios estudios han demostrado, el desarrollo de pequeñas empresas de los sectores industriales y turísticos en regiones agrícolas, representan una respuesta flexible a las nuevas demandas del mercado y a las necesidades de supervivencia en un periodo de crisis prolongada. Como consecuencia, se están expandiendo nuevos tipos de actividades informales como la subcontratación, el trabajo en casa, el alquiler de habitaciones y bares, de forma paralela al 
trabajo agrícola tradicional". Esta idea lleva a hacer dos consideraciones particularmente válidas en las Regiones de Los Ríos y de Los Lagos:

a) Dada la naturaleza y singularidades de los procesos productivos, especialmente de la agricultura ${ }^{6}$, siempre se ha generado un importante porcentaje de empleo informal o subcontratación (no siempre respetando la legislación laboral). Un ejemplo recurrente es el de los trabajadores temporales o "temporeros".

b) En la pequeña agricultura, cuya producción va destinada al autosustento, los miembros de la familia se involucran en la producción.

Ante esta situación, las mujeres y los jóvenes son quienes tienen una mayor predisposición frente a nuevas alternativas productivas y manifiestan una mayor capacidad emprendedora, motivada, fundamentalmente, por la ya referida falta de oportunidades.

\section{EL SECTOR AGRARIO CHILENO: LA CONVIVENCIA DE DOS MUNDOS}

A partir de la segunda mitad de los años ochenta, la economía chilena experimenta un significativo proceso de modernización, internacionalización y crecimiento. La estabilidad macroeconómica, social y política del país, favoreció la inversión privada tanto nacional como extranjera. Paralelamente la población creció de 12,7 a 14 millones de habitantes. Según datos del Instituto Nacional de Estadísticas, el crecimiento poblacional del país se tradujo en un aumento de la población económicamente activa (fuerza de trabajo) de más de un millón de personas, al pasar de 4,5 millones en 1990 a más de 5,8 millones en 2004.

Como consecuencia de los incentivos para la actividad agraria que suponía la favorable evolución del comercio exterior y las mejores condiciones del mercado interno, debido al incremento del poder adquisitivo de la población, se produjo en el sector agropecuario y forestal una importante reasignación de los recursos productivos. El sector cambió sustancialmente: se modificó la estructura de uso del suelo, con la consecuente entrada y salida de productores y empresas; se adecuaron los procesos productivos a los requerimientos de los mercados exteriores; $y$, se elevaron los niveles de competitividad mediante el aumento de la productividad, la reducción de los costes de producción y el establecimiento de menores márgenes de rentabilidad.

Pero la política macroeconómica del país ha tenido también una incidencia decisiva en la evolución del sector agrario chileno, a través del tipo de cambio,

${ }^{6}$ Se hace énfasis en la agricultura porque tradicionalmente la identificamos con el medio rural. Existen localidades rurales cuya actividad productiva básica es otra. En la X Región de Chile, por ejemplo, la pesca o extracción de mariscos tienen una mayor relevancia. 
la política arancelaria y los acuerdos económicos firmados con diversos países de la región. La evolución de estos factores, junto a la fluctuación de los precios internacionales, ha supuesto la necesidad de reconvertir y fortalecer al sector; un sector que, hasta el año 1996, había crecido a una tasa media anual en torno al $5,0 \%$, cifra no muy alejada del $6,2 \%$ que se registró para el conjunto de la economía durante el mismo período ${ }^{7}$. A lo largo de la primera mitad de la década de los noventa, este crecimiento fue impulsado por las exportaciones, que pasaron a representar el $40 \%$ de la producción agraria total, y por la situación de bonanza que se vivía en el mercado interno, activado por la expansión prolongada de los ingresos.

A partir de entonces, se produce una caída y un cambio en la tendencia, a raíz de la crisis económica de los países asiáticos, a los que Chile destina una parte importante de sus exportaciones, y, a juicio de los agricultores, por la política arancelaria y lo poco favorables que resultaban para el sector los acuerdos comerciales firmados por el gobierno (Tratado de Libre Comercio con Méjico y Mercosur, entre otros). A pesar de ello, la participación del sector agrario en el conjunto de la economía del país se ha mantenido prácticamente estable, si bien, continúa conviviendo con problemas de tipo estructural, que atañen principalmente a la situación de la pequeña agricultura y que, por tanto, no pueden solucionarse con planes parciales a corto plazo.

Las principales producciones agrícolas de Chile se presentan en la Tabla 8, en la que se refleja el salto espectacular que, en la última década, ha experimentado el volumen de producción en todos los cultivos. No obstante, dos son los sectores que destacan por haber protagonizado el desarrollo de un sector agrario fuerte y competitivo: el sector forestal y el frutícola.

Tabla 8

Producción de cultivos anuales. (En toneladas)

\begin{tabular}{|l|r|r|r|r|r|r|r|}
\hline \multicolumn{1}{|c|}{ Cultivos } & \multicolumn{1}{c|}{$\mathbf{1 9 9 9 / 0 0}$} & \multicolumn{1}{c|}{$\mathbf{2 0 0 0 / 0 1}$} & \multicolumn{1}{c|}{$\mathbf{2 0 0 1 / 0 2}$} & \multicolumn{1}{c|}{$\mathbf{2 0 0 2 / 0 3}$} & $\mathbf{2 0 0 3 / 0 4}$ & \multicolumn{1}{c|}{$\mathbf{2 0 0 4 / 0 5}$} & \multicolumn{1}{c|}{$\mathbf{2 0 0 8 / 0 9}$} \\
\hline TRIGO & 1.492 .710 & 1.780 .157 & 1.820 .387 & 1.797 .084 & 1.912 .652 & 1.851 .940 & 15.239 .213 \\
\hline AVENA & 247.936 & 344.527 & 416.297 & 488.050 & 538.600 & 357.352 & 3.808 .527 \\
\hline CEBADA & 59.639 & 65.454 & 77.151 & 77.010 & 56.156 & 102.417 & 973.698 \\
\hline CENTENO & 2.396 & 3.735 & 219 & 127 & 127 & & 47.735 \\
\hline MAIZ & 652.019 & 778.498 & 924.211 & 1.189 .729 & 1.320 .606 & 1.507 .766 & 13.579 .209 \\
\hline ARROZ & 135.060 & 43.261 & 141.927 & 140.849 & 119.265 & 116.832 & 946.725 \\
\hline POROTO & 44.274 & 60.447 & 45.043 & 47.909 & 48.712 & 44.597 & 234.193 \\
\hline
\end{tabular}

${ }^{7}$ La Corporación de Fomento (CORFO), argumentando a favor de que la crisis de la agricultura en Chile no tenía un carácter global, señalaba que esta tasa no se había alcanzado jamás en algún decenio histórico con registro. 
Tabla 8 (continuación)

Producción de cultivos anuales. (En toneladas)

\begin{tabular}{|c|c|c|c|c|c|c|c|}
\hline Cultivos & $1999 / 00$ & $2000 / 01$ & $2001 / 02$ & $2002 / 03$ & 2003/04 & $2004 / 05$ & $2008 / 09$ \\
\hline LENTEJA & 1.027 & 1.024 & 632 & 701 & 791 & 1.063 & 7.191 \\
\hline GARBA & 3.247 & 3.689 & 3.024 & 3.085 & 2.941 & 2.919 & 29.888 \\
\hline ARVEJA & 1.805 & 2.466 & 879 & 1.023 & 1.023 & & 22.896 \\
\hline $\mathrm{CHIC}$ & 689 & 133 & 566 & 193 & 193 & & 2.988 \\
\hline PAPA & 38.220 & 1.210 .044 & 1.303.268 & 1.093 .728 & 1.144.170 & 1.115 .736 & 10.813 .492 \\
\hline MARAVILLA & 9.443 & 3.131 & 2.690 & 2.613 & 3.218 & 2.793 & 79.715 \\
\hline RAPS & 7.735 & 67.371 & 2.016 & 19.421 & 22.111 & 41.225 & 439.337 \\
\hline REMOLACHA & 3.112 .407 & 2.882 .980 & 3.191 .807 & 1.953 .306 & 2.278 .303 & 2.597 .771 & 14.206 .681 \\
\hline LUPINO & 41.374 & 37.019 & 31.061 & 43.520 & 52.011 & 63.246 & 737.066 \\
\hline TABACO & 10.291 & 8.534 & 6.976 & 7.713 & 9.321 & 9.850 & 79.495 \\
\hline
\end{tabular}

Fuente: INE (Varios años): Anuario Agropecuario.

Según datos de la Corporación Nacional Forestal (CONAF), el 45\% del territorio chileno está formado por suelos de aptitud forestal, en su mayor parte compuesta por bosque y vegetación nativa (bosque nativo productivo o áreas protegidas). Las plantaciones con fines productivos, en un $70 \%$, corresponden a la especie pino insigne, que es la que sustenta actualmente la industria forestal del país, siguiéndole en importancia las de eucalipto. A pesar de la relevancia de este sector para la economía del país (sus exportaciones representan el 14\% del total y emplea aproximadamente a 100.000 personas), su desarrollo no está exento de polémica, fundamentalmente por las externalidades negativas que genera sobre el medio ambiente, afectando la calidad de vida de los residentes y limitando el desarrollo de otras actividades que, como el turismo, se vinculan estrechamente con los recursos naturales ${ }^{8}$. De manera que nos hallamos ante el sector que más incompatibilidades presenta para el desarrollo de otros (entre ellos el turismo) y que obedecen a las siguientes causas:

- Las externalidades negativas que producen las plantaciones, entre las que se encuentran: el empobrecimiento del suelo, el deterioro del patrimonio paisajístico, o la escasa absorción de mano de obra entre el período de plantación y cosecha.

- La concentración de la propiedad del suelo en manos de grandes empresas forestales, algunas de ellas vinculadas a capital extranjero, situación que ha

\footnotetext{
${ }^{8}$ La instalación de plantas procesadoras de celulosa en la VII y X Región (específicamente en lo que hoy es la Región de Los Ríos), que vierten sus desechos a los cauces de los ríos Itata y Cruces, generando un enorme daño ambiental, han impulsado el nacimiento de movimientos ciudadanos de defensa del medio ambiente.
} 
creado serios conflictos por la "pertenencia de la tierra" con las comunidades indígenas de la VIII, IX y X Regiones, en las que se concentra el potencial forestal del país.

- El inadecuado manejo del bosque nativo, lo que ha significado en algunas zonas explotaciones a "tala rasa" con pérdida irreversible del patrimonio forestal.

Por su parte, el sector frutícola ha sido fundamental en la diversificación de la base exportadora del país. De acuerdo con las cifras del Censo Agropecuario de 1997, la superficie total de frutales alcanzaba 210.917 hectáreas, registrando un incremento del 22,9\% respecto al año 1990. Diez años después, en el Censo Agropecuario y Forestal de 2007 la superficie total de frutales se había incrementado hasta las $324.293,56$ hectáreas, lo que representa un crecimiento del $53,75 \%$. Factores cuyo comportamiento no es fácil de predecir, como la globalización creciente del comercio exterior, la volatilidad de los precios internacionales y el proteccionismo vigente en importantes mercados destino (Unión Europea, Estados Unidos y Brasil), plantean dificultades para que los organismos técnicos del sector efectúen proyecciones fiables. Este hecho dificulta las tareas de gestión y de planificación, así como el diseño e instrumentación de políticas.

Algunos de los elementos estratégicos que la Corporación de Fomento (CORFO) identificaba hace unos años (y que aún están vigentes) para enmarcar el escenario en el que el sector agrario chileno se tendría que desenvolver en las próximas décadas eran los siguientes:

- Mayor grado de competitividad en los mercados internacionales (calidad y precios).

- Apertura creciente e integración en Tratados multilaterales.

- Irrupción en los mercados mundiales de alimentos (oferta y demanda) de los grandes países asiáticos.

- Incremento de los salarios reales del sector agrario.

- Reducción del número de explotaciones y un mayor tamaño medio.

- Disminución de la fuerza de trabajo ocupada en el sector y aumento de la productividad.

- Preocupación por las consideraciones ambientales y la preservación de los recursos naturales.

- Aumento de diversas modalidades asociativas.

- Competitividad multisectorial por el suelo agrícola, lo que implicará la reconversión de su uso (incluyendo usos turísticos, urbano-industrial,...).

Estas líneas estratégicas, que ya en la década de los noventa se comenzaron a manifestar, han sido las premisas sobre las cuales se han basado las actuaciones 
de los organismos públicos vinculados al sector agrario que, con mayor o menor grado de acierto, se han preocupado de la adopción y búsqueda de nuevas alternativas productivas, sobre todo para aquellos medianos y pequeños productores que, dedicados consuetudinariamente a trabajar en sectores tradicionales (cultivo de cereales, ganadería y producción de leche), hoy tienen escasa viabilidad para seguir con el mismo esquema productivo.

Como ha podido observarse, el sector agrario chileno se mueve entre dos mundos. En él conviven desde empresas transnacionales (subsectores forestal, frutícola, vitivinícola y forestal, fundamentalmente), pasando por otras de mediano tamaño, con moderna tecnología y capacidad de innovación, hasta pequeños propietarios, inmersos en una agricultura de subsistencia, con escaso capital y sin tecnología. Esta agricultura campesina o familiar es la que presenta serios problemas de viabilidad, puesto que muchas de las explotaciones obtienen rendimientos muy bajos ${ }^{9}$. Las características de este pequeño productor rural chileno se pueden resumir en función de los siguientes parámetros (Correa, 1995; Amtmann, 1999):

- Escasa dotación de tierra, generalmente de calidad y ubicación marginal, con precarios derechos de tenencia.

- Limitados recursos de capital, situación que agrava las dificultades de accesibilidad a los insumos y medios modernos de producción.

- Abundante mano de obra, predominantemente de carácter familiar, en gran parte subempleada, cuya oferta para trabajar fuera del predio complementa sus ingresos de subsistencia.

- Explotaciones de carácter mixto con cultivos asociados y cría de animales para intentar asegurar la subsistencia del grupo familiar, minimizando los riesgos.

- Bajo rendimiento de sus explotaciones y baja calidad de los productos obtenidos que, además, están sujetos a grandes pérdidas por una deficiente conservación y comercialización.

- Producción para su subsistencia, ofreciendo al mercado sus excedentes sin añadir valor a sus productos, vendiendo en forma individual y a bajos precios.

- Rendimiento laboral y desempeño empresarial se ven afectados por sus precarias condiciones ambientales y de salud.

\footnotetext{
${ }^{9}$ Se estima que 177.000 familias dedicadas a esta actividad son pobres; es decir, disponen de muy poca superficie, muestran los mayores índices de pobreza rural y cualquier alternativa de desarrollo pasa por dotarlos de infraestructuras básicas.
} 
- Poca confianza en la organización comunitaria, siendo escasa e ineficiente la cooperación entre ellos para afrontar unidos sus problemas, aun cuando se muestran solidarios con el grupo al que pertenecen.

- Dificultades de acceso a la tecnología moderna y a los servicios del Estado que sólo llegan a una minoría de ellos de forma parcial y descoordinada.

- Bajo nivel de capacitación que les impide utilizar racionalmente los recursos disponibles, adoptar tecnologías apropiadas y organizarse, usando servicios y recursos externos a sus predios y comunidades. La Tabla 9 muestra que en las pequeñas unidades prediales se concentra la mayor cantidad de personas sin ningún nivel formal de educación $(64,7 \%)$. Esta variable resulta clave para mejorar el bienestar socioeconómico de las poblaciones más postergadas.

Tabla 9

Productores agropecuarios sin ningún nivel de educación según tamaño de la explotación

\begin{tabular}{|l|c|}
\hline \multicolumn{1}{|c|}{ Tamaño de la explotación } & Número de personas \\
\hline Menores de 1 ha. & 5.039 \\
\hline De 1 a menos de 5 ha. & 10.155 \\
\hline De 5 a menos de 10 ha & 5.644 \\
\hline De 10 a menos de 20 ha. & 5.157 \\
\hline De 20 a menos de 50 ha & 3.798 \\
\hline De 50 a menos de 100 ha & 1.390 \\
\hline De 100 a menos de 200 ha. & 556 \\
\hline De 200 a menos de 500 ha & 267 \\
\hline De 500 a menos de 1000 ha. & 104 \\
\hline De 1000 a menos de 2000 ha. & 53 \\
\hline De 2000 ha y más & 50 \\
\hline Total país & 32.213 \\
\hline
\end{tabular}

Fuente: INE: Censo Agropecuario, 2007.

Pero, tal vez, lo más importante, sea recalcar que si algo caracteriza al pequeño agricultor o campesino chileno es su situación de pobreza e incluso indigencia, problema que se agudiza por la nula capacidad de respuesta que ofrecen las formas tradicionales de producción. Las palabras del Presidente de la Comisión Nacional de Superación de la Pobreza, Alberto Echegaray, que se recogen a continuación, dan idea de la situación en que se encuentra la pobreza rural en Chile: "El suelo es el recurso natural que sostiene la vida del 
campesino: es decir, el campesino vive de los ingresos, tiende a sobreexplotar la tierra aumentando la superficie cultivada, la frecuencia de las siembras o introduce ganado, lo que trae mayor explotación sobre las tierras empobrecidas. La sobreexplotación trae erosión y empobrecimiento del suelo, por lo tanto, menor rendimiento y menores ingresos. Para equiparar los ingresos, es preciso sobreexplotar aún más el recurso, el cual más pronto que tarde colapsa y la pobreza se torna irremediable. El resultado es la emigración a la ciudad de personas con gran dificultad de adaptación para sobrevivir. Esto es lo que algunos han llamado la espiral de la ecopobreza".

El desarrollo de la agricultura familiar campesina es la base de la estrategia de superación de la pobreza rural. Si bien nadie duda de lo imprescindible que resulta la ampliación y mejora de programas sociales que ataquen directamente la pobreza e indigencia rural, también existe la certeza de que el dinamismo de las actividades económicas del sector rural, fundamentalmente de la agricultura, conforman un elemento determinante para la superación de la pobreza en el campo. En esta misma perspectiva se inscribe la aportación de las actividades no agrícolas, que para una parte importante de la población rural y de la agricultura familiar campesina constituyen un complemento significativo de sus ingresos ${ }^{10}$.

\section{EL TURISMO RURAL COMO ALTERNATIVA PARA LA DIVERSIFICACIÓN PRODUCTIVA}

El turismo rural y sus encadenamientos, está siendo considerado de forma cada vez más recurrente en los análisis de diferentes organismos internacionales, gobiernos nacionales y locales, instituciones de fomento, organizaciones no gubernamentales, entre otros, como un factor potencialmente importante para la diversificación de la economía rural y para el empleo rural no agrícola.

El turismo rural en Chile surge en 1996 a partir de la propuesta "La visión de ruralidad 2010" siendo el resultado de un ciclo de talleres para la formulación de propuestas de políticas públicas para la reducción de la pobreza rural, organizado por la Red de Cooperación Institucional para Zonas de Pobreza Rural (Prorural) dependiente del Instituto Nacional de Desarrollo Agropecuario (INDAP). A partir de ese mismo año, en la Región de Los Lagos se fomenta, por este mismo Instituto, la creación de una oferta de hospedajes rurales en la ribera del lago Llanquihue. El resultado de esta iniciativa piloto permitió replicar este modelo de desarrollo en otras zonas rurales de la Región,

${ }^{10}$ Así se recoge en el Acta de Acuerdos de la Mesa para el Desarrollo de la Agricultura Familiar Campesina, reunida en la ciudad de Santiago el 8 de octubre de 2001. 
y así surgieron las Redes de Agroturismo de Chiloé, Tres Islas, Futronhue, Las Gaviotas y Corral, por citar las más antiguas.

Como ya se ha señalado, en toda la macroregión sur de Chile, la existencia de un paisaje definido por el agua y la selva valdiviana (bosque siempre verde), dan forma a un espacio natural rural rico en flora y fauna, que tradicionalmente ha constituido un área interesante para la demanda turística. En esta zona, y más específicamente en la ribera sur del citado lago Llanquihue, se concentran importantes centros turísticos (Frutillar y Puerto Varas), que de una u otra manera incitaron a los agricultores locales, muchos de ellos herederos de tradiciones y costumbres alemanas, a abrir sus casas al turista, incorporando el agroturismo, incluso cuando aún no se había definido claramente el concepto, al mercado turístico. Por lo señalado y por las características productivas (región ganadera y lechera por excelencia), es en este sector donde la oferta de turismo rural, y en particular de agroturismo, es más homogénea y alcanza un buen nivel de competitividad.

En otros sectores de la zona, en una etapa de desarrollo más incipiente, se han implementado otros proyectos gestionados por familias campesinas, que muestran una oferta más heterogénea en cuanto a composición y calidad y en algunos casos presentan evidentes problemas de sostenibilidad en el tiempo, variados son los factores que atentan contra la viabilidad de estas propuestas. Estos contrastes que se presentan en este territorio conducen a tener presente que las propuestas que se elaboren deben considerar que existen emprendimientos que se enfrentan a desafíos diferentes. La creación de redes, fomentadas por la institucionalidad pública, ha sido la respuesta para disminuir las brechas y buscar, principalmente, algunas economías de escala en la parte organizacional, administrativa y promoción de la oferta de los asociados.

Actualmente el turismo rural se encuentra en un proceso de consolidación de la oferta, con la creación de Redes de Agroturismo que han permitido generar economías de escala en lo que se refiere a cuestiones de organización administrativa y de promoción a sus asociados.

\subsection{Situación competencial chilena en materia de turismo rural}

Sí se tiene en cuenta que la población rural en Chile, según datos del Censo de 2002, es de 2.026.322 personas, es posible señalar que el volumen potencial de beneficiarios de los programas y proyectos de desarrollo de turismo rural, que impulsan tanto organismos del sector público como privado tendrán un impacto significativo si se planifican adecuadamente y surgen a partir de iniciativas participativas, en las que el producto final resulte del consenso de las propias comunidades.

En Chile, ante la creciente demanda de este tipo de turismo, tanto las autoridades públicas y empresarios agrícolas, como los pobladores y pequeños 
productores rurales, han visto en el turismo rural una nueva oportunidad de actividad productiva que contribuya a disminuir, al menos en parte, la migración rural, la falta de oportunidades de empleo, la crisis del sector agrario tradicional y el creciente desaliento que se palpa entre las comunidades rurales. En consecuencia, los planes de desarrollo regional, provincial, o comunal incluyen cada vez con mayor frecuencia la identificación de algunos programas o proyectos vinculados a la actividad turística con los que se propone impulsar el desarrollo del medio rural.

Es amplía la gama de factores que explican el interés creciente que han puesto distintos organismos, fundamentalmente estatales, en fomentar las actividades turísticas que tienen como escenario al medio rural, pero entre todos ellos destaca la posibilidad de facilitar la generación de "negocios" que complementen los ingresos producidos por las explotaciones agrarias, sobre todo de los sectores más pobres, contribuyendo con ello a superar el problema de la pobreza y a garantizar la igualdad de oportunidades.

Desde la segunda mitad de la década de los noventa, las acciones emprendidas por distintos organismos públicos, se traducen en buscar nuevas alternativas de desarrollo, que vayan más allá de las directamente relacionadas con las formas tradicionales de explotación o producción del suelo rural. De manera, que se comienza a incorporar el tema de los servicios $\mathrm{y}$, particularmente, el de la prestación de servicios de turismo rural.

Los organismos del sector público que directa o indirectamente desarrollan programas o proyectos relacionados con el turismo rural se recogen en la Tabla 10.

Tabla 10

Principales instituciones públicas vinculadas al turismo rural

\begin{tabular}{|l|l|l|}
\hline \multicolumn{1}{|c|}{ Institución } & \multicolumn{1}{|c|}{ Dependencia } & \multicolumn{1}{c|}{ Cobertura } \\
\hline $\begin{array}{l}\text { Corporación de Fomento } \\
\text { (CORFO) }\end{array}$ & Ministerio de Planificación & $\begin{array}{l}\text { Nacional } \\
\text { Todos los sectores de la } \\
\text { economía }\end{array}$ \\
\hline $\begin{array}{l}\text { Fondo de Solidaridad e } \\
\text { Inversión Social (FOSIS) }\end{array}$ & Ministerio de Agricultura & $\begin{array}{l}\text { Nacional } \\
\text { Grupos vulnerables de la } \\
\text { sociedad. }\end{array}$ \\
\hline $\begin{array}{l}\text { Instituto Nacional de } \\
\text { Desarrollo Agropecuario } \\
\text { (INDAP) }\end{array}$ & Ministerio de Economía & $\begin{array}{l}\text { Nacional } \\
\text { Sector agropecuario }\end{array}$ \\
\hline $\begin{array}{l}\text { Servicio Nacional de } \\
\text { Turismo (SERNATUR) }\end{array}$ & $\begin{array}{l}\text { Parte de las trece unidades } \\
\text { territoriales en que se divide } \\
\text { política y administrativamente el } \\
\text { país. Su autoridad máxima es el } \\
\text { Intendente designado por el } \\
\text { Presidente de la República. }\end{array}$ & $\begin{array}{l}\text { Nacional } \\
\text { Sector turismo }\end{array}$ \\
\hline \multicolumn{2}{|l|}{} \\
\hline
\end{tabular}


Tabla 10 (continuación)

Principales instituciones públicas vinculadas al turismo rural

\begin{tabular}{|l|l|l|}
\hline \multicolumn{1}{|c|}{ Institución } & \multicolumn{1}{|c|}{ Dependencia } & \multicolumn{1}{c|}{ Cobertura } \\
\hline \multirow{5}{*}{ Gobiernos Regionales } & $\begin{array}{l}\text { Son las unidades territoriales } \\
\text { menores en que se divide } \\
\text { política y administrativamente el } \\
\text { país (342 comunas). La } \\
\text { autoridad comunal es el Alcalde, } \\
\text { que es elegido por votación } \\
\text { popular }\end{array}$ & $\begin{array}{l}\text { Regional } \\
\text { Gobierno y administración } \\
\text { de la Región }\end{array}$ \\
\hline Gobiernos Locales & & $\begin{array}{l}\text { Comunal } \\
\text { Gobierno y administración } \\
\text { de la comuna }\end{array}$ \\
\hline
\end{tabular}

Fuente: Elaboración propia.

En el nivel nacional, no existe una política específica dirigida a tal fin, pero distintos organismos de cobertura nacional actúan en función de sus programas y objetivos particulares, influyendo sobre aspectos relacionados con el turismo rural. Estas instituciones tienen un carácter centralizado y operativamente funcionan a través de sus oficinas regionales y/o provinciales. Pueden intervenir en las regiones a través de las Secretarias Ministeriales (SEREMI), correspondientes al Ministerio del que dependen, y más directamente a través de las unidades que han creado al efecto en su estructura organizacional. En algunos casos, estas instituciones tienen una cobertura territorial bastante amplia, como sucede con el Instituto Nacional de Desarrollo Agropecuario (INDAP), el Fondo de Solidaridad e Inversión Social (FOSIS) y la Corporación de Fomento (CORFO). En otros, es más limitada, como por ejemplo, la Fundación de Innovación Agraria (FIA) o el Servicio Nacional de Turismo (SERNATUR).

A escala local, son los Planes Comunales de Desarrollo (PLADECO) los que, de una u otra manera, deberían considerar el turismo, dependiendo de la vocación productiva de la localidad. La escasez de recursos económicos y humanos cualificados limita las posibilidades de esta herramienta.

Además de la acción pública, actúan también como gestores o promotores de proyectos de turismo rural campesino distintos organismos privados, fundaciones, corporaciones, organizaciones no gubernamentales, universidades,... Ahora bien, no siempre la forma de actuar de estos agentes responde a una estrategia que explícitamente identifique al turismo rural como su eje.

Este numeroso grupo de instituciones públicas y privadas, actuando simultáneamente sobre un mismo territorio en pro del turismo rural, produce, en muchas ocasiones, problemas de descoordinación. 


\subsection{Turismo y mujer en el medio rural chileno: Los problemas de género}

No se puede soslayar, en este ambiente de pobreza y de marginación, el importante papel que desempeñan las mujeres. Más allá de reconocerles su fortaleza para sostener el concepto de "familia", aún en las condiciones socioeconómicas más desfavorables, en muchas zonas rurales de Chile se están constituyendo en los "agentes del cambio".

Las mujeres rurales, genéricamente, desempeñan tres roles: reproducción familiar; tareas económico-productivas; $\mathrm{y}$, labores de carácter comunitario. La forma en que se desenvuelven en estos roles está en función de las condiciones étnicas, socioeconómicas y geográficas. Su trabajo, en general, se califica como "invisible" y no lo recogen las estadísticas oficiales. En el medio rural, la falta de infraestructura y equipamiento social genera una gran dependencia de la familia en torno a la mujer, por lo que si hay algo consensuado es que las mujeres son un factor de cohesión del territorio y verdaderos agentes dinamizadores (López, 1999).

Enfrentadas a limitadas opciones, el turismo, que en este medio se asocia a un trabajo propio del ámbito tradicional femenino y que requiere el desarrollo de actividades de carácter más doméstico, se ha transformado en la vía por la cual la mujer comienza a pasar a desarrollar una actividad económica formal y a jerarquizar su trabajo. Las mujeres y los jóvenes, frente al escaso papel que han desempeñado hasta el momento, principalmente por factores de orden cultural, son más abiertos frente a nuevas iniciativas. Generalmente, son ellas las que hacen suya y se entusiasman con la idea de abrir sus casas a los visitantes y, con ello, contribuir al ingreso familiar. Por el contrario, el hombre es más reticente y sólo a medida que ve en ella una actividad económicamente rentable se incorpora. Las palabras de una mujer de Melipehuco (Región de La Araucanía) así lo demuestran: "al principio mi marido no compartía la idea; hoy está entusiasmado construyendo la segunda cabaña, y mi hijo es el responsable de las excursiones a caballo" $" 11$.

\section{CONSIDERACIONES FINALES}

A pesar de la favorable evolución que han registrado en Chile los indicadores macroeconómicos en los últimos años, continúa siendo una asignatura pendiente, al margen de los esfuerzos realizados por los tres últimos gobiernos democráticos, el déficit que sigue manifestando el modelo económico en cuanto a la equidad en la distribución de los ingresos (Chile es uno de los países de Iberoamérica con la peor distribución del ingreso). Esta situación repercute considerablemente en la agricultura familiar o campesina ya que en

${ }^{11}$ Entrevista realizada a una participante del proyecto de turismo rural de la Asociación de Municipios Precordilleranos de la Región de La Araucanía, en la localidad de Melipehuco. 
algunas zonas del país está suponiendo su virtual desaparición.

Los problemas estructurales y las perspectivas poco favorables para el sector agrario tradicional, han sido la premisa sobre la cual se han basado las actuaciones de los organismos públicos que, con mayor o menor grado de acierto, se han preocupado de la adopción y búsqueda de nuevas alternativas productivas, entre ellas el turismo rural, sobre todo para aquellos medianos y pequeños productores que, dedicados consuetudinariamente, a trabajar en sectores tradicionales (cultivo de cereales, ganadería y producción de leche) hoy tienen escasa viabilidad.

La "pequeña agricultura campesina o familiar", que tiene su origen en el campo, que mantiene una relación hombre-tierra muy estrecha, con características culturales y sociales bien definidas, que incorpora la familia al proceso productivo, y que se asocia con los índices de pobreza de las zonas rurales, se encuentra en estos momentos ante un futuro incierto y cada vez más constreñido.

Por ello, desde la segunda mitad de la década de los noventa, las acciones emprendidas por distintos organismos públicos se traducen en buscar nuevas alternativas de desarrollo, que vayan más allá de las directamente relacionadas con las formas tradicionales de producción, comenzando a incorporarse la prestación de servicios de turismo rural.

Ahora bien, estas nuevas iniciativas que se están produciendo en el medio rural, ya sea espontánea y/o planificadamente, provocan cambios en la estructura productiva que modifican las características ocupacionales de la población. La mujer y los jóvenes son quienes están manifestando una mayor predisposición para adoptar nuevas alternativas productivas, como el turismo rural.

El desafío es que las riquezas y potencialidades del espacio rural chileno sean integralmente utilizadas para la mejora de la calidad de vida de los residentes locales, teniendo en cuenta que las estrategias o acciones que se desarrollen en el futuro con este fin deberán contar con su activa participación.

\section{REFERENCIAS BIBLIOGRÁFICAS}

ALVAYAY, V.; ROJAS, M.; y, VILLARROEL, K. (2004): Programa de Fomento Productivo para los Sectores más Pobres de la Pequeña Agricultura. Tesis para optar al Magíster de Gerencia y Políticas Públicas. Universidad Adolfo Ibáñez. Mimeo. 
AMTMANN, C. y FERNANDEZ F. (Editores) (1981): "Comunicación y Desarrollo rural". Publicación del Instituto de Ciencias Históricas y Sociales, a través de su «Programa Centro de Sociología del Desarrollo Rural», Santiago. Universidad Austral de Chile.

AMTMANN, C.F.; MÚJICA, B. y VERA (1998): "Pequeña agricultura en la Región de Los Lagos", Chile. Edita Universidad Austral de Chile pp. 9-26.

BERDEGUÉ, J.; REARDON, T.; y, ESCOBAR, G. (2003): "Empleo e Ingreso Rural no Agrícola en América Latina y El Caribe". Intercambios, № 29, Año 3.

BLANCO PORTILLO, P.R. y BENAYAS DEL ÁLAMO, J. (1994): "El turismo como motor de desarrollo rural. Análisis de los proyectos de turismo subvencionados por LEADER". Revista de Estudios Agrosociales, № 169.

CONSTABEL, S.; OYARZÚN, E. y SZMULEWICZ, P. (2005): Agroturismo en Chile: Sistematización de la experiencia nacional. Fundación para la Innovación Agraria y Universidad Austral de Chile. Santiago. 320 páginas.

CORREA, L.M. (1995): Diagnóstico de la situación productiva y caracterización socioeconómica de los pequeños productores incorporados a Indap, pertenecientes a la Comuna de Corral. Tesis Doctoral. Facultad de Ciencias Veterinarias. Universidad Austral de Chile, Valdivia. Mimeo.

FONDO DE SOLIDARIDAD E INVERSIÓN SOCIAL (FOSIS) (1997). Boletín Regional del Fosis, $N^{\circ}$ 2. Santiago. Ministerio de Planificación y Cooperación.

FONDO DE SOLIDARIDAD E INVERSIÓN SOCIAL (FOSIS) (1998): Programa de Desarrollo Productivo Rural. Bases del Quinto Concurso. Ministerio de Planificación y Cooperación, Santiago, Mayo.

GALÁN, B. (1994): Participación campesina para una agricultura sostenible en países de América Latina. Roma. Organización de las Naciones Unidas para la Agricultura y la Alimentación.

HERNÁNDEZ SÁNCHEZ, A. (1989): "La población y ordenación del territorio en Castilla y León". Anales de Estudios Económicos y Empresariales, № 4. Valladolid. Universidad de Valladolid.

HUERTA, M.A. (1989): Otro agro para Chile. La historia de la Reforma Agraria en el proceso social y político. Santiago. Ediciones Chile América CESOC.

INSTITUTO NACIONAL DE ESTADÍSTICAS (INE) (1992): Censo de población y Vivienda 1992. Jefe Departamento de Atención al Usuario y Difusión, INE. Santiago.

INSTITUTO NACIONAL DE ESTADÍSTICAS (INE) (2002): "Resultados. Censo de Vivienda y Población". Jefe Departamento de Atención al Usuario y Difusión, INE. Santiago.

LÓPEZ BERNAL, R.E. (1999): Programas de fomento productivo, interacción de actores y desarrollo económico local. Caso del Programa Fosis de apoyo a la microempresa en la Región de Valparaíso. Mimeo. 
MINISTERIO DE PLANIFICACIÓN (MIDEPLAN) (1990): Dimensiones y características de la pobreza, según Casen. División de Planificación, Estudios e Inversión, Santiago. 
